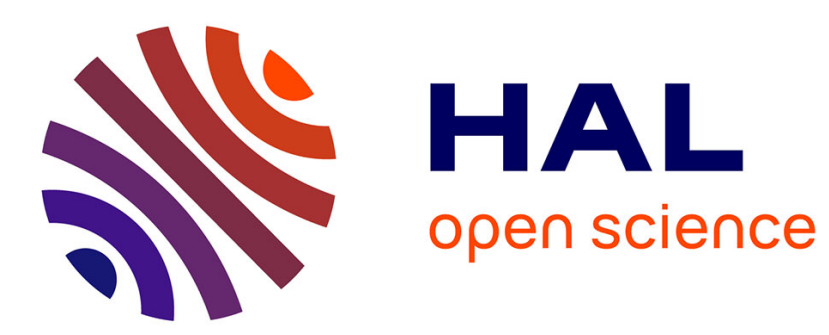

\title{
Conventional and advanced MRI in multiple sclerosis
}

\author{
C. Louapre
}

\section{To cite this version:}

C. Louapre. Conventional and advanced MRI in multiple sclerosis. Revue Neurologique, 2018, 174

(6), pp.391 - 397. 10.1016/j.neurol.2018.03.009 . hal-01840049

\section{HAL Id: hal-01840049 \\ https: / hal.sorbonne-universite.fr/hal-01840049}

Submitted on 16 Jul 2018

HAL is a multi-disciplinary open access archive for the deposit and dissemination of scientific research documents, whether they are published or not. The documents may come from teaching and research institutions in France or abroad, or from public or private research centers.
L'archive ouverte pluridisciplinaire HAL, est destinée au dépôt et à la diffusion de documents scientifiques de niveau recherche, publiés ou non, émanant des établissements d'enseignement et de recherche français ou étrangers, des laboratoires publics ou privés. 


\title{
Conventional and advanced MRI in multiple sclerosis
}

\section{Louapre ${ }^{a, b, *}$}

${ }^{a}$ Inserm UMR S 1127, CNRS UMR 7225, Institut du Cerveau et de la Moelle épinière, ICM, Hôpital de la PitiéSalpêtrière, Sorbonne Université, UPMC Paris 06, Paris, France

b Département de neurologie, CIC Neurosciences, Hôpital Pitié-Salpêtrière, AP-HP, Bâtiment ICM, 75013 Paris, France

\begin{abstract}
A B S T R A C T
Magnetic resonance imaging (MRI) plays a central role in the management of patients with multiple sclerosis (MS). T2-weighted/FLAIR lesions have been included in the diagnostic criteria since 2001, and the importance of the technology has been expanded in each successive revision of the McDonald criteria. While the typical focal hyperintense lesions seen on T2 and FLAIR sequences in several areas of the central nervous system are key features for MS diagnosis, they can also be used to monitor disease activity, particularly in asymptomatic patients, and to evaluate therapeutic responses. The development of new lesions, particularly in medullary and infratentorial locations, is a strong predictor of longterm disability and risk of evolution to a secondary-progressive phase. Yet, changes in T2 lesion volume are poor predictors of subsequent disease evolution in many cases, a situation often referred to as the "clinicoradiological paradox". Nevertheless, advanced MRI techniques allow quantification of several pathological processes in vivo and offer insights into MS pathophysiology beyond white matter lesions. By investigating what is happening beneath the visible surface of MS pathology, these techniques not only help to unravel the clinicoradiological paradox, but also provide early measures of functional and structural tissue abnormalities before the advent of irreversible neurodegeneration.
\end{abstract}

\section{Introduction}

Magnetic resonance imaging (MRI) has been established as a key tool in the diagnosis of multiple sclerosis (MS), and remains central in the algorithm to assess dissemination in space (DIS) and dissemination in time (DIT) in the upcoming 2017 MS diagnosis criteria [1,2]. However, despite its high sensitivity for MS diagnosis (which can be challenged in cases lacking typical appearances of T2-weighted lesions),

\footnotetext{
* Correspondence. CIC Neurosciences, Hôpital Pitié-Salpêtrière, Bâtiment ICM, 75013 Paris, France.

E-mail address: celine.louapre@aphp.fr.

Abbreviations: ADC, apparent diffusion coefficient; CDMS, clinically definite multiple sclerosis; CIS, clinically isolated syndrome; CSF, cerebrospinal fluid; DIR, double inversion recovery; GM, gray matter; MRI, magnetic resonance imaging; MS, multiple sclerosis; MTR, magnetization transfer ratio; NAWM, normal-appearing white matter; RIS, radiologically isolated syndrome; RRMS, relapsing-remitting multiple sclerosis; SPMS, secondary-progressive multiple sclerosis; tNAA, total N-acetylaspartate; tCr, total creatine; WM, white matter.
} 
conventional MRI cannot explain the wide heterogeneity of clinical outcomes. Nevertheless, the present review emphasizes the importance of conventional MRI for MS diagnosis and prognosis, and the potential of non-conventional MRI to allow visualization of its various pathophysiological mechanisms, including subpial demyelination, normal-appearing white matter (NAWM), microstructural damage and neurodegeneration.

\section{Conventional MRI: a diagnostic tool for MS}

The clinical relevance of MS lesion detection by conventional T2-weighted fluid-attenuated inversion recovery (FLAIR) sequences on brain MRI, and T2 and short-tau inversion recovery (STIR) sequences on medullary MRI, was outlined by the incorporation of imaging criteria into the international McDonald criteria for MS in 2001, as well as its subsequent 2005 and 2010 revisions. Since 2010, the diagnosis of MS can be assessed by a single MRI in patients presenting with a clinically isolated syndrome (CIS), as DIT criteria can be fulfilled solely by the concomitant presence of (asymptomatic) lesions with and without gadolinium enhancement [3]. The recently published McDonald 2017 criteria for MS diagnosis [2] have only been slightly modified regarding MRI criteria for DIS and DIT, as symptomatic and asymptomatic MRI lesions can now be considered in determining both these designations. Moreover, cortical lesions can now be used to meet MRI criteria for DIS, whereas only juxtacortical lesions were considered in the 2010 revision.

To increase the sensitivity and specificity of MS lesion detection by MRI, standardized acquisition protocols have been recommended by two expert groups, namely, the Observatoire français de la sclérose en plaques (OFSEP) in France [4] and the European research network Magnetic Resonance Imaging in MS (MAGNIMS) [5].

Yet, despite these efforts towards the use of standardized MRI protocols, several caveats should be borne in mind. The use of high magnetic field strengths [such as 3 Tesla (T)] increases sensitivity for detecting white matter (WM) lesions compared with lower magnetic field strengths (for example, $1.5 \mathrm{~T}$ ). Conversely, the specificity of MS lesions diagnosed by T2/FLAIR hyperintensities relies on the expertise of neuroradiologists and/or neurologists and can be challenged, especially in older subjects who have concomitant cerebral small vessel disease. In a recent multicenter study of 110 misdiagnosed patients, the main alternative diagnosis of MS was migraine (22\%), a condition associated with WM hyperintensities and subclinical brain infarcts [6]. Moreover, the sensitivity and specificity for detecting spinal cord lesions were lower compared with brain lesions, mainly due to partial volume effects, respiratory movements and artefacts in surrounding tissue.

\section{Non-conventional MRI can refine MS diagnosis}

When neuropathological studies described the perivenous distribution of MS lesions, it led to the use of MRI sequences able to detect vascular structures in vivo, such as T2*-weighted and susceptibility-weighted imaging (SWI). Using a combination of these sequences together with conventional FLAIR, several studies have described the "central vein sign" and suggested that the presence of this sign can increase specificity in detecting MS lesions (Fig. 1). In a study of 33 MS patients by Kilsdonk et al. [7], combining FLAIR and T2* sequences at $7 \mathrm{~T}$ determined that $78 \%$ of the lesions were located around a vessel whereas, in small vessel disease and neuromyelitis optica spectrum disorder (NMOSD), the proportion of lesions associated with a vascular structure is significantly lower (usually $<40 \%$ ). However, a recent consensus statement from the North American Imaging in Multiple Sclerosis (NAIMS) Cooperative pointed out the difficulties in defining the central vein sign radiologically and the need for further investigation of its clinical value, as the sign can also be observed in various other pathological conditions, albeit at lower rates than in MS [8].

Detection of the cortical lesions known to arise at early MS stages and predominantly in progressive phases of the disease is also unlikely using conventional sequences. However, over the past decade, the use of double inversion recovery (DIR) sequences has improved their detection, such that the presence of cortical lesions has been integrated into the 2017 revised MRI criteria for DIS [1]. However, DIR can only detect around $18 \%$ of cortical lesions based on radiological and neuropathological investigations, which means that most subpial lesions are undetected [9], whereas the use of 7-T acquisitions markedly improves sensitivity for detecting both cortical and intracortical subpial lesions [10]. Unfortunately, 7T MRI acquisitions are not yet implemented in daily clinical practice because of the limited number of scanners and high cost of such acquisitions.

\section{Conventional T2 lesions as a prognostic marker}

Epidemiological studies of large MS cohorts have emphasized the importance of WM lesions to predict the risk of clinical conversion from radiologically isolated syndrome (RIS) to CIS and from CIS to clinically definite MS (CDMS). In the cohort study from Barcelona [11], which included 1058 CIS patients followed for a mean duration of 17 years, the presence of at least one lesion on brain MRI predicted conversion to CDMS vs patients with no MRI lesions with a hazard ratio (HR) of 5.1. This HR, however, jumped to 11.3 when the baseline MRI displayed $>10$ lesions, independently of the presence of oligoclonal bands (OCBs) in cerebrospinal fluid (CSF).

While longitudinal cohorts of RIS patients are scarce, they can point out the crucial importance of WM brain lesion numbers in predicting conversion to CIS, together with the potent predictive value of the presence of medullary lesions $[12,13]$. However, the presence of asymptomatic spinal cord lesions was the strongest predictor [odds ratio (OR): 128 on multivariate analysis] of conversion from RIS to CIS or primary-progressive (PP) MS in 71 patients with RIS [13].

Results from the longitudinal London cohort study of 166 CIS patients followed for 15 years highlighted the importance of early imaging markers to predict risk of evolution to 


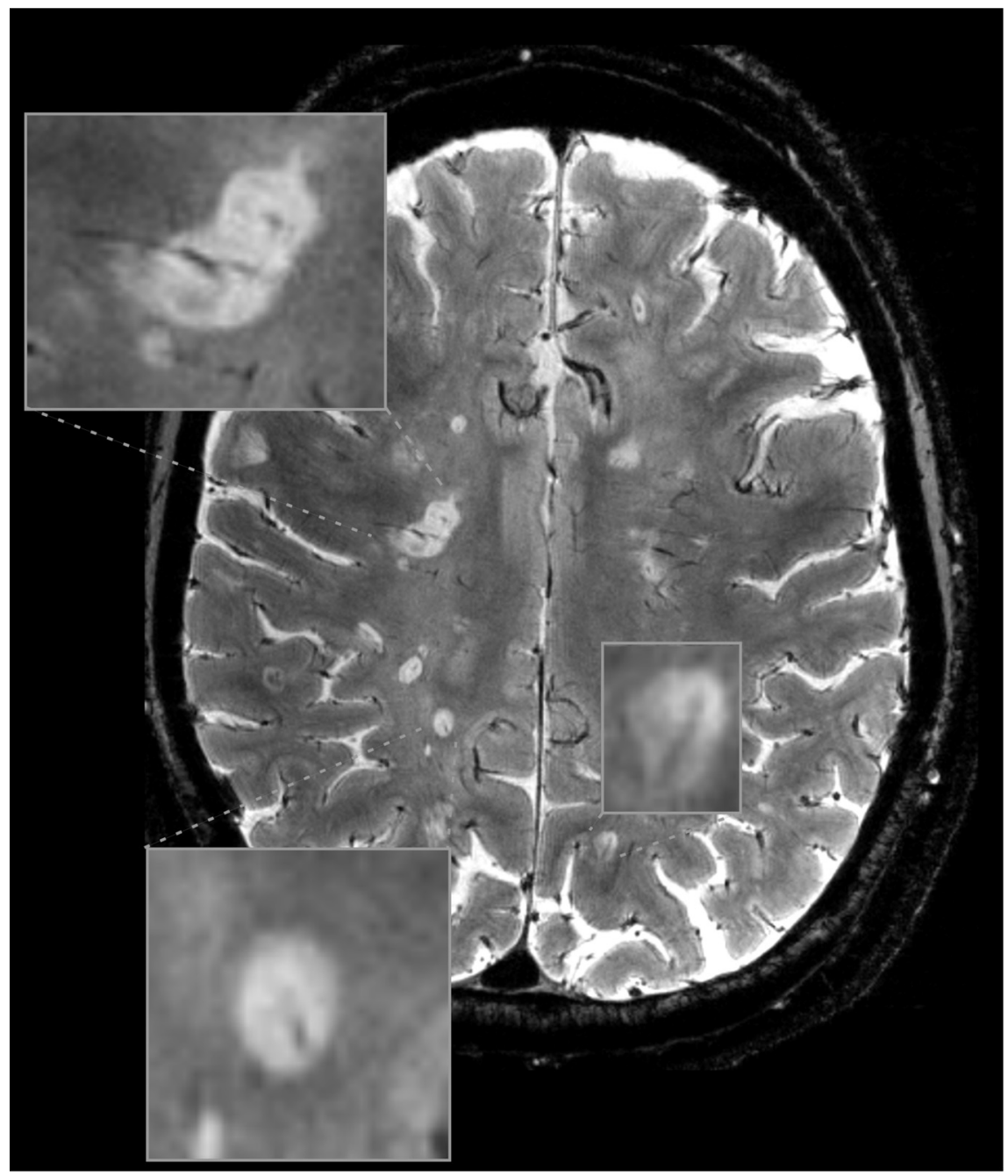

Fig. 1 - The central vein sign on T2*-weighted imaging acquired at 7 Tesla.

secondary-progressive (SP) MS during long-term follow-up [14]. Indeed, the development of T2 lesions within the first year of CIS increased the risk of long-term SPMS (OR: 2-4), while the presence of medullary and/or infratentorial lesions at baseline predicted the risk of long-term SPMS (OR: 5-7). In that cohort study, the combined presence of at least two lesions with gadolinium enhancement at baseline and the appearance of new medullary or infratentorial lesions during the first year of follow-up could predict, by up to $85 \%$, the long-term risk of SPMS.

In many studies, however, the volume and/or number of WM lesions and their evolution correlated poorly with increases in disability, and the prognostic value seemed to be even less evident at later stages and in progressive forms of MS [15]. This has been dubbed the "clinicoradiological paradox" (Fig. 2), which can be explained by a variety of factors not accessible by conventional MRI (including diversity of WM lesion subtypes, gray matter [GM] lesions and NAWM pathology). The paradox is further accentuated by the lack of reliable measures of neurological disability to assess MS progression [16].
Nevertheless, several MRI studies have provided clues to unravel the clinicoradiological paradox. First, when taking into account the location of lesions, WM lesions in specific clinically relevant tracts is strongly associated with subsequent disability, as measured by the Expanded Disability Status Scale (EDSS), in both relapsing and progressive forms of MS $[17,18]$. Second, numerous studies exploring functional connectivity using blood-oxygen-level-dependent (BOLD) functional MRI (fMRI) acquisitions, whether at rest or during an activation task, have provided evidence that brain reorganization processes can significantly counteract clinical expressions of tissue injury. In fact, from the earliest stages of CIS, increased resting-state connectivity has been demonstrated by several large-scale brain networks which, thereafter, further decreased to the levels seen in controls several years after disease onset [19]. In a cohort of patients with severe early-stage cognitive impairment, decreased functional connectivity has been found in key networks, such as the default-mode and attentional networks, compared with controls and patients with no cognitive impairment [20]. Similarly, during a cognitive task with fMRI, Audoin et al. [21] 


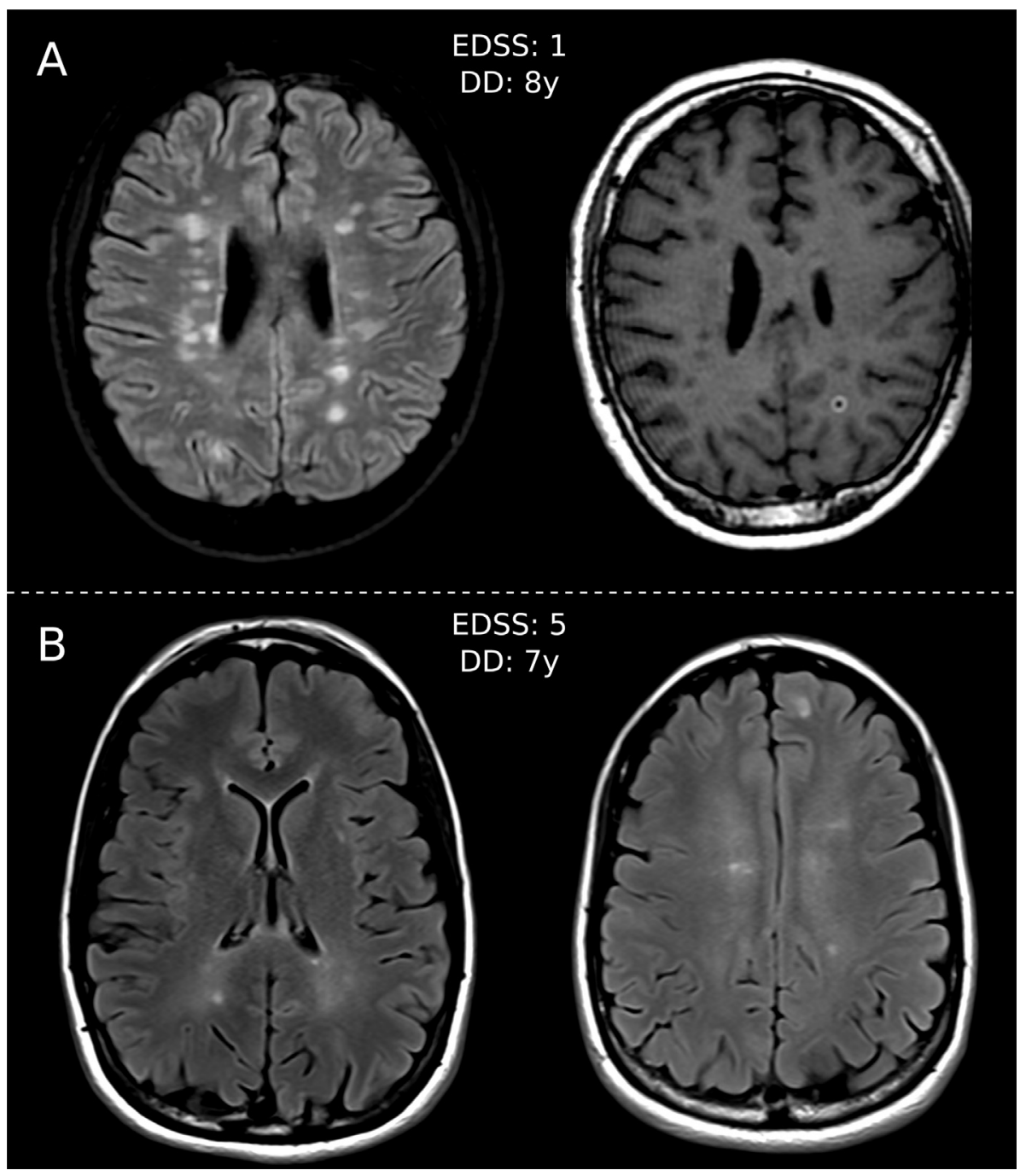

Fig. 2 - The clinicoradiological paradox in multiple sclerosis: (A) high brain lesion load in a patient with minor signs on examination, an Expanded Disability Status Scale (EDSS) score of 1 and disease duration (DD) of 8 years; and (B) low lesion load in a patient with marked disability (walking distance of $250 \mathrm{~m}$, attentional dysfunction), and an EDSS score of 5 and DD of 7 years. Diffuse abnormalities of white matter beyond focal lesions are seen on fluid-attenuated inversion recovery (FLAIR) sequences; medullary MRI revealed one cervical lesion.

demonstrated increased cortical activation in CIS subjects compared with controls in the absence of any cognitive deficit, while a study by Bonnet et al. [22] provided further insight into the limitations of such overactivation during more demanding cognitive tasks and in patients with poorer cognitive performances.

\section{Using MRI to monitor disease activity and predict therapeutic responses}

To date, monitoring disease activity has somewhat exclusively been achieved by longitudinal evaluations of WM lesion volume, although the detection of new or enlargening lesions can be challenging. Automatic lesion segmentation software, aiming to co-register acquisitions at different time points to enhance sensitivity for detecting new T2 lesions, is not yet available for routine clinical use. Despite this, it is now well established that the accumulation of new or enlargening T2 or gadolinium-enhancing lesions is the strongest predictor of subsequent relapses. Two meta-analyses of 23 and 31 published randomized, double-blind, placebo-controlled clinical trials, respectively, in relapsing-remitting (RR) MS demonstrated that the effect of therapy on accumulation of WM lesions during the first 6 months can accurately predict the therapeutic effect on relapses over longer follow-up durations (12-24 months) [23,24]. This suggests that MRI metrics could be used as primary endpoints in clinical trials to shorten their duration and lower their costs.

\section{MRI to monitor disease-modifying therapy safety}

Progressive multifocal leukoencephalopathy (PML) represents the most severe adverse event with MS disease-modifying 


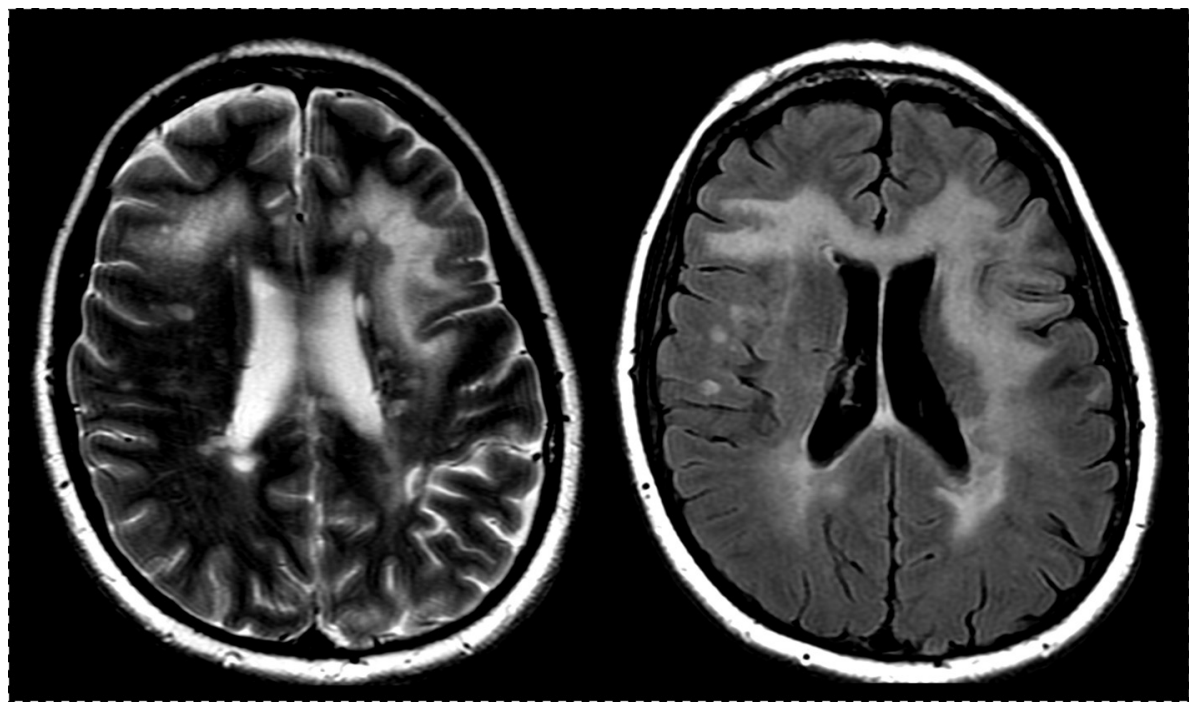

Fig. 3 - Typical MRI features of progressive multifocal leukoencephalopathy in a multiple sclerosis patient treated with natalizumab: (left) T2-weighted axial sequence; and (right): fluid-attenuated inversion recovery (FLAIR) axial sequence.

therapies (DMTs), especially natalizumab, a humanized monoclonal antibody against integrin $\alpha 4$ adhesion molecules (Fig. 3). Early detection of asymptomatic PML is of crucial importance, as diagnostic delay has been associated with negative outcomes and lower survival rates [25]. Therefore, MRI should be performed at least once a year in patients treated by natalizumab, and even more frequently - up to every 3 months - in patients at higher PML risk [26].

Low sensitivity for detecting new PML lesions and the possible misdiagnosis of new MS lesions are two challenging factors when monitoring MS treatment safety. A study by Wijburg et al. [27] identified several radiological characteristics to differentiate PML from MS lesions. These included the presence of punctate T2 lesions, cortical GM involvement, juxtacortical WM involvement, ill-defined and mixed lesion borders favoring both GM and WM, lesions $>3 \mathrm{~cm}$ in size and contrast enhancement.

\section{Advanced MRI for beyond WM lesions: imaging MS pathophysiology}

Imaging metrics focused on macroscopic WM lesions are of limited prognostic value for MS disability and progression, whereas volumetric measures derived from conventional T1weighted acquisitions have proved to be significant predictors of disability progression in longitudinal studies. The GM portion decreases more rapidly in SPMS than in either RRMS or CIS [28] while, in PPMS, the decrease in brain volume over 2 years is an independent predictor of disability progression over 10 years [29], and atrophy of key cerebral structures (corpus callosum, neocortex) is a strong surrogate marker of cognitive deterioration [30,31].

Volumetric measures are still not available in everyday clinical practice, as post-processing requires computational time, technological assessment and linear optimization. Also, GM structural segmentation processes can be challenging in the presence of WM lesions, although it is now widely implemented in large-scale multicenter clinical trials and should become a prominent outcome measure in early-phase clinical trials in progressive MS [32].

However, advanced MRI techniques can provide additional measures to reflect the dynamic evolution of damage within lesions and tissue damage outside of lesions. These techniques also have the potential to improve the prognostic value of MRI metrics while also providing more insight into the pathophysiology of the disease.

Contrast agents to MRI sequences reflecting myelin tissue contents and WM bundle microstructure like diffusion tensor imaging (DTI) and magnetization transfer imaging (MTI) have been widely used over the past decade, and have emphasized the importance of diffuse abnormalities in NAWM. Recently, using magnetization transfer ratio (MTR) imaging, Liu et al. [33] described a gradient of periventricular abnormalities in NAWM that was more pronounced in SPMS than in RRMS. In early MS, this periventricular gradient also proved to be a predictor of conversion to CDMS and EDSS scores 5 years later [34]. This highlights the potential role of CSF immunemediated factors in determining diffuse neurodegeneration in MS outside of focal WM lesions, and its potential role in long-term disability progression.

Other brain compartmental abnormalities are difficult to evaluate using standard MRI acquisitions. Subpial demyelination, which has been described in neuropathology cases [35] and appears to be extensive, involving up to $90 \%$ of the cortex in progressive MS, can be more accurately quantified using ultra-high-field MRI [36]. Two studies, from Nielsen et al. [37] and Harrison et al. [38], demonstrated that cortical lesions are the strongest correlates of physical and cognitive outcomes in MS (intracortical and leukocortical lesions, respectively), independently of conventional MRI metrics 
such as WM lesions and volumetric measures. A study using quantitative $\mathrm{T} 2^{*}$ measures, reflecting cortical tissue myelin and iron contents, found that subtle tissue abnormalities extend beyond the visible cortical lesions, particularly in SPMS [39].

Recently, using a combination of 7-T T2* and 3-T T1/T2 myelin-sensitive imaging with neurite orientation dispersion and density imaging (NODDI) for assessing microstructural myelin, axonal and dendrite integrity, Granberg et al. [40] demonstrated, in a cohort of 26 MS patients with disease durations $<2$ years, that cortical demyelination happens very early in the disease course. In fact, these tissue changes are likely to precede cortical thinning, which was not detected at this disease stage.

Metrics reflecting neuronal dysfunction before neuronal loss may prove useful for capturing early potentially reversible mechanisms of neurodegeneration that may be susceptible to neuroprotective therapeutic strategies. When ${ }^{23} \mathrm{Na}$ MRI was used to evaluate GM sodium accumulation in 58 MS patients with disease durations $\leq 10$ years, Maarouf et al. [41] found that total GM sodium concentration was a better predictor of cognitive impairment than GM atrophy.

Bodini et al. [42] used diffusion-weighted magnetic resonance spectroscopy (DW-MRS) to investigate neuroaxonal damage and energy dysregulation in a cohort study of 25 MS patients. This method offers insight into functional and microstructural measures in the neuroaxonal compartment [such as diffusivity of total N-acetylaspartate (tNAA)] as well as measures related to cell energy metabolism [such as diffusivity of total creatine (tCr)]. The authors found, in patients vs controls, a reduction of tNAA apparent diffusion coefficient (ADC) in GM, most likely as a reflection of neuroaxonal damage, as well as a reduction of tCr ADC in NAWM and GM, most likely reflecting energy dysregulation.

\section{Conclusion}

In clinical practice, MRI is a powerful tool for diagnosing MS, and monitoring disease activity and therapeutic responses, via T2-weighted WM lesion volume. However, by confining evaluation of MS patients to WM lesions only, most of the pathophysiological mechanisms that determine long-term disability and negative outcomes are either overlooked or underestimated. While advanced MRI techniques have become more commonly used for secondary outcomes in clinical trials, additional efforts should now focus on making these techniques more widely available and feasible for everyday clinical practice to achieve personalized patient care adapted to the various pathological mechanisms in play in MS.

\section{Disclosure of interest}

Dr Louapre has received compensation for travel grants, consulting services and speaker honoraria from Biogen, Genzyme, Merck Serono, Novartis and Teva, but none related to the present work.
[1] Cohen JA. 2017 proposed revisions to the McDonald diagnostic criteria for multiple sclerosis. MSPARIS2017 49. Mult Scler J 2017;23(Suppl. 3):8-84.

[2] Thompson AJ, Banwell BL, Barkhof F, Carroll WM, Coetzee T, Comi G, et al. Diagnosis of multiple sclerosis: 2017 revisions of the McDonald criteria. Lancet Neurol 2018;17(2):162-73.

[3] Polman CH, Reingold SC, Banwell B, Clanet M, Cohen JA, Filippi M, et al. Diagnostic criteria for multiple sclerosis: 2010 revisions to the McDonald criteria. Ann Neurol 2011;69(2):292-302.

[4] Cotton F, Kremer S, Hannoun S, Vukusic S, Dousset V. Imaging Working Group of the Observatoire Francais de la Sclerose en P. OFSEP, a nationwide cohort of people with multiple sclerosis: consensus minimal MRI protocol. J Neuroradiol 2015;42(3):133-40.

[5] Rovira A, Wattjes MP, Tintore M, Tur C, Yousry TA, Sormani MP, et al. Evidence-based guidelines: MAGNIMS consensus guidelines on the use of MRI in multiple sclerosis-clinical implementation in the diagnostic process. Nat Rev Neurol 2015;11(8):471-82.

[6] Solomon AJ, Bourdette DN, Cross AH, Applebee A, Skidd PM, Howard DB, et al. The contemporary spectrum of multiple sclerosis misdiagnosis: a multicenter study. Neurology 2016;87(13):1393-9.

[7] Kilsdonk ID, Lopez-Soriano A, Kuijer JP, de Graaf WL, Castelijns JA, Polman CH, et al. Morphological features of MS lesions on FLAIR* at 7T and their relation to patient characteristics. J Neurol 2014;261(7):1356-64.

[8] Sati P, Oh J, Constable RT, Evangelou N, Guttmann CR, Henry $R G$, et al. The central vein sign and its clinical evaluation for the diagnosis of multiple sclerosis: a consensus statement from the North American Imaging in Multiple Sclerosis Cooperative. Nat Rev Neurol 2016;12(12):714-22.

[9] Seewann A, Kooi EJ, Roosendaal SD, Pouwels PJ, Wattjes MP, van der Valk $P$, et al. Postmortem verification of MS cortical lesion detection with 3D DIR. Neurology 2012;78(5):302-8

[10] Pitt D, Boster A, Pei W, Wohleb E, Jasne A, Zachariah CR, et al. Imaging cortical lesions in multiple sclerosis with ultra-high-field magnetic resonance imaging. Arch Neurol 2010;67(7):812-8.

[11] Tintore M, Rovira A, Rio J, Otero-Romero S, Arrambide G, Tur C, et al. Defining high, medium and low impact prognostic factors for developing multiple sclerosis. Brain 2015;138(Pt 7):1863-74.

[12] Lebrun C, Bensa C, Debouverie M, Wiertlevski S, Brassat D, de Seze J, et al. Association between clinical conversion to multiple sclerosis in radiologically isolated syndrome and magnetic resonance imaging, cerebrospinal fluid, and visual evoked potential: follow-up of 70 patients. Arch Neurol 2009;66(7):841-6.

[13] Okuda DT, Mowry EM, Cree BA, Crabtree EC, Goodin DS, Waubant E, et al. Asymptomatic spinal cord lesions predict disease progression in radiologically isolated syndrome. Neurology 2011;76(8):686-92.

[14] Brownlee WJ, Altmann D, Miszkiel KA, Prados F, Eshaghi A, Ourselin S, et al. New spinal cord and infratentorial lesions in early relapse-onset MS are predictive of secondary progressive disease course after 15 years. MSPARIS2017 101. Mult Scler J 2017;23(Suppl. 3):8-84.

[15] Li DK, Held U, Petkau J, Daumer M, Barkhof F, Fazekas F, et al. MRI T2 lesion burden in multiple sclerosis: a plateauing relationship with clinical disability. Neurology 2006;66(9):1384-9. 
[16] Chard D, Trip SA. Resolving the clinico-radiological paradox in multiple sclerosis. F1000Res 2017;6:1828.

[17] Dalton CM, Bodini B, Samson RS, Battaglini M, Fisniku LK, Thompson AJ, et al. Brain lesion location and clinical status 20 years after a diagnosis of clinically isolated syndrome suggestive of multiple sclerosis. Mult Scler 2012;18(3):322-8.

[18] Bodini B, Battaglini M, De Stefano N, Khaleeli Z, Barkhof F, Chard D, et al. T2 lesion location really matters: a 10 year follow-up study in primary progressive multiple sclerosis. J Neurol Neurosurg Psychiatry 2011;82(1):72-7.

[19] Roosendaal SD, Schoonheim MM, Hulst HE, Sanz-Arigita EJ, Smith SM, Geurts JJ, et al. Resting state networks change in clinically isolated syndrome. Brain 2010;133(Pt 6):1612-21.

[20] Louapre C, Perlbarg V, Garcia-Lorenzo D, Urbanski M, Benali $\mathrm{H}$, Assouad R, et al. Brain networks disconnection in early multiple sclerosis cognitive deficits: an anatomofunctional study. Hum Brain Mapp 2014;35(9):4706-17.

[21] Audoin B, Ibarrola D, Ranjeva JP, Confort-Gouny S, Malikova I, Ali-Cherif A, et al. Compensatory cortical activation observed by fMRI during a cognitive task at the earliest stage of MS. Hum Brain Mapp 2003;20(2):51-8.

[22] Bonnet MC, Allard M, Dilharreguy B, Deloire M, Petry KG, Brochet B. Cognitive compensation failure in multiple sclerosis. Neurology 2010;75(14):1241-8.

[23] Sormani MP, Bonzano L, Roccatagliata L, Cutter GR, Mancardi GL, Bruzzi P. Magnetic resonance imaging as a potential surrogate for relapses in multiple sclerosis: a meta-analytic approach. Ann Neurol 2009;65(3):268-75.

[24] Sormani MP, Bruzzi P. MRI lesions as a surrogate for relapses in multiple sclerosis: a meta-analysis of randomised trials. Lancet Neurol 2013;12(7):669-76.

[25] Vermersch P, Kappos L, Gold R, Foley JF, Olsson T, Cadavid D, et al. Clinical outcomes of natalizumabassociated progressive multifocal leukoencephalopathy. Neurology 2011;76(20):1697-704.

[26] McGuigan C, Craner M, Guadagno J, Kapoor R, Mazibrada G, Molyneux P, et al. Stratification and monitoring of natalizumab-associated progressive multifocal leukoencephalopathy risk: recommendations from an expert group. J Neurol Neurosurg Psychiatry 2016; 87(2):117-25.

[27] Wijburg MT, Witte BI, Vennegoor A, Roosendaal SD, Sanchez E, Liu Y, et al. MRI criteria differentiating asymptomatic PML from new MS lesions during natalizumab pharmacovigilance. J Neurol Neurosurg Psychiatry 2016;87(10):1138-45.

[28] Fisher E, Lee JC, Nakamura K, Rudick RA. Gray matter atrophy in multiple sclerosis: a longitudinal study. Ann Neurol 2008;64(3):255-65.

[29] Khaleeli Z, Ciccarelli O, Manfredonia F, Barkhof F, Brochet $\mathrm{B}$, Cercignani $\mathrm{M}$, et al. Predicting progression in primary progressive multiple sclerosis: a 10-year multicenter study. Ann Neurol 2008;63(6):790-3.
[30] Amato MP, Portaccio E, Goretti B, Zipoli V, Battaglini M, Bartolozzi ML, et al. Association of neocortical volume changes with cognitive deterioration in relapsing-remitting multiple sclerosis. Arch Neurol 2007;64(8):1157-61.

[31] Granberg T, Martola J, Bergendal G, Shams S, Damangir S, Aspelin P, et al. Corpus callosum atrophy is strongly associated with cognitive impairment in multiple sclerosis: results of a 17-year longitudinal study. Mult Scler 2015;21(9):1151-8.

[32] Moccia M, de Stefano N, Barkhof F. Imaging outcome measures for progressive multiple sclerosis trials. Mult Scler 2017;23(12):1614-26.

[33] Liu Z, Pardini M, Yaldizli O, Sethi V, Muhlert N, WheelerKingshott CA, et al. Magnetization transfer ratio measures in normal-appearing white matter show periventricular gradient abnormalities in multiple sclerosis. Brain 2015;138(Pt 5):1239-46.

[34] Brown JW, Pardini M, Brownlee WJ, Fernando K, Samson RS, Prados Carrasco F, et al. An abnormal periventricular magnetization transfer ratio gradient occurs early in multiple sclerosis. Brain 2017;140(2):387-98.

[35] Kutzelnigg A, Lucchinetti CF, Stadelmann C, Bruck W, Rauschka H, Bergmann M, et al. Cortical demyelination and diffuse white matter injury in multiple sclerosis. Brain 2005;128(Pt 11):2705-12.

[36] Mainero C, Benner T, Radding A, van der Kouwe A, Jensen $\mathrm{R}$, Rosen $\mathrm{BR}$, et al. In vivo imaging of cortical pathology in multiple sclerosis using ultra-high field MRI. Neurology 2009;73(12):941-8.

[37] Nielsen AS, Kinkel RP, Madigan N, Tinelli E, Benner T, Mainero C. Contribution of cortical lesion subtypes at 7T MRI to physical and cognitive performance in MS. Neurology 2013;81(7):641-9.

[38] Harrison DM, Roy S, Oh J, Izbudak I, Pham D, Courtney S, et al. Association of cortical lesion burden on 7-T magnetic resonance imaging with cognition and disability in multiple sclerosis. JAMA Neurol 2015;72(9):1004-12.

[39] Louapre C, Govindarajan ST, Gianni C, Langkammer C, Sloane JA, Kinkel RP, et al. Beyond focal cortical lesions in MS: an in vivo quantitative and spatial imaging study at 7T. Neurology 2015;85(19):1702-9.

[40] Granberg T, Fan Q, Treaba CA, Ouellette R, Herranz E, Mangeat $\mathrm{G}$, et al. In vivo characterization of cortical and white matter neuroaxonal pathology in early multiple sclerosis. Brain 2017;140(11):2912-26.

[41] Maarouf A, Audoin B, Pariollaud F, Gherib S, Rico A, Soulier E, et al. Increased total sodium concentration in gray matter better explains cognition than atrophy in MS. Neurology 2017;88(3):289-95.

[42] Bodini B, Branzoli F, Poirion E, Garcia-Lorenzo D, Didier M, Maillart E, et al. Dysregulation of energy metabolism in multiple sclerosis measured in vivo with diffusionweighted spectroscopy. Mult Scler 2017 [1352458517698249]. 